\title{
AN APPLICATION OF MULTISTATE RELIABILITY THEORY TO AN OFFSHORE GAS PIPELINE NETWORK
}

\author{
BENT NATVIG, bent@math.uio.no \\ Department of Mathematics, University of Oslo, \\ P.O. Box 1053 Blindern, N-0316 Oslo, Norway \\ HANS W.H. MØRCH, hans-wilhelm.morch@lu.hio.no \\ Faculty of Education, Oslo University College, \\ Pilestredet 52, N-0167 Oslo, Norway
}

\begin{abstract}
The basic multistate reliability theory was developed in the eighties and the beginning of the nineties, replacing traditional reliability theory where the system and the components are always described as functioning or failed. In Natvig et al. [10] the theory was applied to an electrical power generation system for two nearby oilrigs, where the amounts of power that may possibly be supplied to the two oilrigs are considered as system states. However, there is still a need for several convincing case studies demonstrating the practicability of the generalizations introduced. In the present paper the theory is applied to the Norwegian offshore gas pipeline network in the North Sea, as of the end of the eighties, transporting gas to Emden in Germany. The system state depends on the amount of gas actually delivered, but also to some extent on the amount of gas compressed mainly by the compressor component closest to Emden.
\end{abstract}

Keywords: availabilities, unavailabilities

\section{Introduction and basic definitions}

The basic multistate reliability theory was developed in the eighties and the beginning of the nineties, replacing traditional reliability theory where the system and the components are always described as functioning or failed. A review of the early development in this area is given in Natvig [8]. In Natvig et al. [10] the theory was applied to an electrical power generation system for two nearby oilrigs, where the 
amounts of power that may possibly be supplied to the two oilrigs are considered as system states. However, more than fifteen years later, there is still a need for several convincing case studies demonstrating the practicability of the generalizations introduced. In the present paper the theory is applied to the Norwegian offshore gas pipeline network in the North Sea, as of the end of the eighties, transporting gas to Emden in Germany. The system state depends on the amount of gas actually delivered, but also to some extent on the amount of gas compressed mainly by the compressor component closest to Emden.

Let $S=\{0,1, \ldots, M\}$ be the set of states of the system; the $M+1$ states representing successive levels of performance ranging from the perfect functioning level $M$ down to the complete failure level 0 . Furthermore, let $C=\{1, \ldots, n\}$ be the set of components and $S_{i}(i=1, \ldots, n)$ the set of states of the $i$ th component. We claim $\{0, M\} \subseteq S_{i} \subseteq S$. Hence, the states 0 and $M$ are chosen to represent the endpoints of a performance scale that might be used for both the system and its components. Let $x_{i}(i=1, \ldots, n)$ denote the state or performance level of the $i$ th component and $\boldsymbol{x}=\left(x_{1}, \ldots, x_{n}\right)$. It is assumed that the state, $\phi$, of the system is given by the structure function $\phi=\phi(\boldsymbol{x})$. In this paper we consider the following type of multistate systems for which a series of results can be derived.

Definition 1.1 A system is a multistate monotone system (MMS) iff its structure $\phi$ satisfies:

(i) $\phi(\boldsymbol{x})$ is non-decreasing in each argument

(ii) $\phi(\mathbf{0})=0$ and $\phi(\boldsymbol{M})=M \quad(\mathbf{0}=(0, \ldots, 0), \boldsymbol{M}=(M, \ldots, M))$.

The first assumption roughly says that improving one of the components cannot harm the system, whereas the second says that if all components are in the complete failure (perfect functioning) state, then the system is in the complete failure (perfect functioning) state.

In what follows $\boldsymbol{y}<\boldsymbol{x}$ means $y_{i} \leq x_{i}$ for $i=1, \ldots, n$, and $y_{i}<x_{i}$ for some $i$.

Definition 1.2 Let $\phi$ be the structure function of an $M M S$ and let $j \in\{1, \ldots, M\}$. $A$ vector $\boldsymbol{x}$ is said to be a minimal path (cut) vector to level $j$ iff $\phi(\boldsymbol{x}) \geq j$ and $\phi(\boldsymbol{y})<j$ for all $\boldsymbol{y}<\boldsymbol{x}(\phi(\boldsymbol{x})<j$ and $\phi(\boldsymbol{y}) \geq j$ for all $\boldsymbol{y}>\boldsymbol{x})$.

Definition 1.3 The performance process of the $i$ th component $(i=1, \ldots, n)$ is a stochastic process $\left\{X_{i}(t), t \in[0, \infty)\right\}$, where for each fixed $t \in[0, \infty) X_{i}(t)$ is a random variable which takes values in $S_{i}$. The joint performance process for the components $\{\boldsymbol{X}(t), t \in[0, \infty)\}=\left\{\left(X_{1}(t), \ldots, X_{n}(t)\right), t \in[0, \infty)\right\}$ is the corresponding vector stochastic process. The performance process of an MMS with structure function $\phi$ is a stochastic process $\{\phi(\boldsymbol{X}(t)), t \in[0, \infty)\}$, where for each fixed $t \in[0, \infty), \phi(\boldsymbol{X}(t))$ is a random variable which takes values in $S$. 
Definition 1.4 The performance processes $\left\{X_{i}(t), t \in[0, \infty)\right\}, i=1, \ldots, n$ are independent in the time interval $I$ iff, for any integer $m$ and $\left\{t_{1}, \ldots, t_{m}\right\} \subset I$ the random vectors $\left\{X_{1}\left(t_{1}\right), \ldots, X_{1}\left(t_{m}\right)\right\}, \ldots,\left\{X_{n}\left(t_{1}\right), \ldots, X_{n}\left(t_{m}\right)\right\}$ are independent.

Definition 1.5 Let $j \in\{1, \ldots, M\}$. The availability, $h_{\phi}^{j(I)}$ and the unavailability, $g_{\phi}^{j(I)}$ to level $j$ in the time interval $I$ for an MMS with structure function $\phi$ are given by

$$
h_{\phi}^{j(I)}=P[\phi(\boldsymbol{X}(s)) \geq j \forall s \in I], \quad g_{\phi}^{j(I)}=P[\phi(\boldsymbol{X}(s))<j \forall s \in I] .
$$

Note that $h_{\phi}^{j(I)}+g_{\phi}^{j(I)} \leq 1$, with equality for the case $I=[t, t]$. In Funnemark and Natvig [3] and Natvig [9] bounds for $h_{\phi}^{j(I)}$ and $g_{\phi}^{j(I)}$ are arrived at, based on corresponding information on the multistate components, generalizing bounds given in Natvig [7] for the case $M=1$. The components are assumed to be maintained and interdependent. In Natvig [11] sufficient conditions are given for some of these bounds to be strict, and also exact, contributing to the understanding of the nature of the bounds and to their applicability. It is the aim of this paper to give such bounds for our offshore gas pipeline network.

In the latter paper it is also shown that for the case where the performance processes of the components are independent in $I$, no additional assumption that each of these is associated in $I$, is needed to establish any strict or non-strict bounds.

Neither Esary \& Proschan [2] and Natvig [7] treating the binary case nor Funnemark \& Natvig [3] and Natvig [9] treating the multistate case were aware of this. Accordingly, this was not taken into account in the case study considered in Natvig et al. [10], but it is in the present paper.

\section{An offshore gas pipeline network}

The offshore gas pipeline network treated in this paper is the most complex of the ones considered in Mørch [6]. It constitutes the main parts of the network in the North Sea, as of the end of the eighties, transporting gas to Emden in Germany. The network along with its modules are given in Figure 2.1. As can be seen from this figure the network consists altogether of 32 components and 7 non-trivial modules. $a_{1}$ and $a_{2}$ are pipelines from the production field at Statfjord, $c_{1}$ from the Heimdal and Troll fields, $c_{2}$ from the Sleipner field and finally $e$ from the Ekofisk field. All these oil and gas fields are in the Norwegian sector of the North Sea west of southern Norway. $k$ is the pipeline from what is called H7 to Emden. The compressor components of the network are $f_{1}, h_{1}$ and $j_{1}$. 

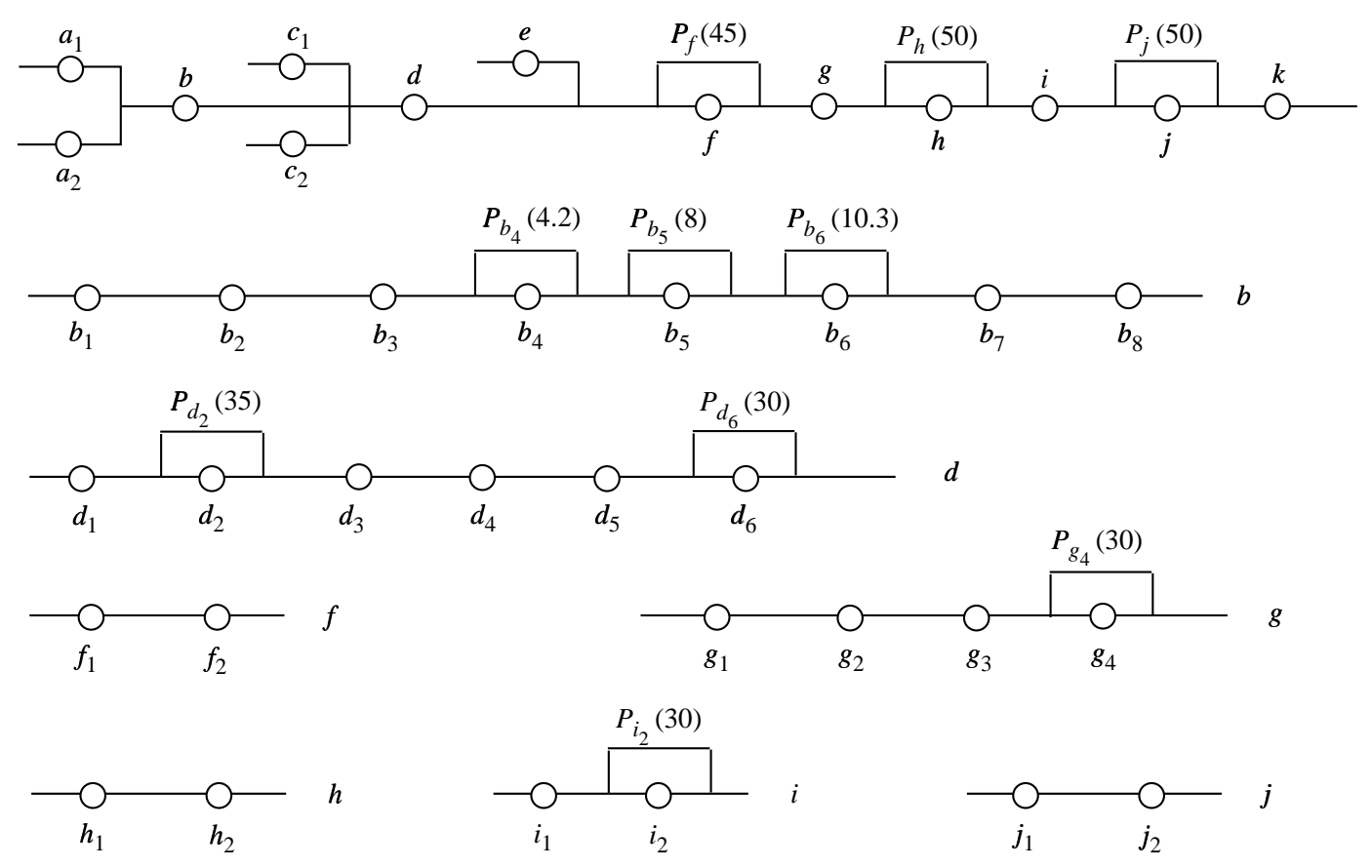

Figure 2.1. Gas pipeline network with corresponding modules $b, d, f, g, h, i, j$.

There are 10 passages in the network all supposed to function perfectly. For instance, $P_{f}(45)$ is the passage of module $f$ having a capacity of $45 \mathrm{MSm}^{3} / d$ (million standard cubic metres per day). Similarly, $P_{b_{4}}(4.2)$ is the passage of component $b_{4}$ having a capacity of $4.2 \mathrm{MSm}^{3} / d$. The module passages $P_{f}, P_{h}$ and $P_{j}$ are used whenever the corresponding modules cannot transport and compress the incoming amounts of gas, by transporting, within their capacities, the surplus amount of gas. The 7 component passages are only used when the corresponding components are not in the perfect functioning state, chosen somewhat arbitrarily to be $M=16$.

The gas pipeline from Ekofisk to Emden being part of this network is called Norpipe. Today there are two more pipelines, Europipe 1 and Europipe 2, providing Norwegian offshore gas to Emden. In addition Zeepipe provides gas to Zeebrugge in Belgium and Franpipe gas to Dunkerque in France. Hence, the total Norwegian offshore gas pipeline network of today is more complex than the one considered in the present paper.

Let us look closer at the compressor components and start with $f_{1} . f_{1}$ consists of four compressors each with a capacity of transporting and compressing $11 \mathrm{MSm}^{3} / d$. The states of $f_{1}$ are defined in Table 2.1. Since the expected maintenance time for a compressor is assumed longer than the expected repair time, it is seen from the table that a failed compressor leads to a higher component state than a maintained one. Furthermore, we assume that at most three compressors can be used at the same time implying that the maximum capacity of $f_{1}$ is $33 \mathrm{MSm}^{3} / d$, which is achieved when 


\begin{tabular}{|c|c|c|c|}
\hline State & $\begin{array}{c}\text { The number of } \\
\text { operative compressors }\end{array}$ & $\begin{array}{c}\text { The number of } \\
\text { failed compressors }\end{array}$ & $\begin{array}{c}\text { The number of } \\
\text { maintained compressors }\end{array}$ \\
\hline 0 & 0 & 3 & 1 \\
\hline 1 & 0 & 4 & 0 \\
\hline 2 & 1 & 2 & 1 \\
\hline 3 & 1 & 3 & 0 \\
\hline 4 & 2 & 1 & 1 \\
\hline 5 & 2 & 2 & 0 \\
\hline 14 & 3 & 0 & 0 \\
\hline 15 & 3 & 1 & 0 \\
\hline 16 & 4 & 0 & \\
\hline
\end{tabular}

Table 2.1. The states of compressor component $f_{1}$.

the component state is 14,15 or 16 . The compressor components $h_{1}$ and $j_{1}$ both consists of three compressors each with a capacity of transporting and compressing 4.5 $\mathrm{MSm}^{3} / d$. The states of $h_{1}$ and $j_{1}$ are defined in Table 2.2.

\begin{tabular}{|c|c|c|c|}
\hline State & $\begin{array}{c}\text { The number of } \\
\text { operative compressors }\end{array}$ & $\begin{array}{c}\text { The number of } \\
\text { failed compressors }\end{array}$ & $\begin{array}{c}\text { The number of } \\
\text { maintained compressors }\end{array}$ \\
\hline 0 & 0 & 2 & 1 \\
\hline 1 & 0 & 3 & 0 \\
\hline 2 & 1 & 1 & 1 \\
\hline 3 & 1 & 2 & 0 \\
\hline 14 & 2 & 0 & 1 \\
\hline 15 & 2 & 1 & 0 \\
\hline 16 & 3 & 0 & 0 \\
\hline
\end{tabular}

Table 2.2. The states of compressor components $h_{1}$ and $j_{1}$.

Now at most two compressors can be used at the same time implying that the maximum capacity of both $h_{1}$ and $j_{1}$ is $9 \mathrm{MSm}^{3} / d$, which is achieved when the component state is 14,15 or 16 .

For the remaining 29 components gas can only be transported when the component is in the perfect functioning state 16. The corresponding transport capacities are given i Table 2.3 .

\begin{tabular}{|c|c|c|c|c|c|c|c|c|c|c|c|c|c|c|}
\hline$a_{1}$ & $a_{2}$ & $b_{1}$ & $b_{2}$ & $b_{3}$ & $b_{4}$ & $b_{5}$ & $b_{6}$ & $b_{7}$ & $b_{8}$ & $c_{1}$ & $c_{2}$ & $d_{1}$ & $d_{2}$ & $d_{3}$ \\
\hline 5 & 20 & 20 & 20 & 16 & 16 & 16 & 16 & 16 & 16 & 48 & 42 & 50 & 50 & 50 \\
\hline$d_{4}$ & $d_{5}$ & $d_{6}$ & $\bar{e}$ & $f_{2}$ & $g_{1}$ & $q_{2}$ & $g_{3}$ & $g_{4}$ & $h_{2}$ & $i_{1}$ & $i_{2}$ & $j_{2}$ & $k$ & \\
\hline 50 & 50 & 50 & 11 & 33 & 59 & 59 & 59 & 59 & 9 & 59 & 59 & 9 & 59 & \\
\hline
\end{tabular}

Table 2.3. Transport capacities in $\mathrm{MSm}^{3} / d$ for the remaining 29 components. 
For all these components the set of states is of the form $\{0,1,2, \ldots, r, 16\}$, where $0 \leq r<16$. The states $0,1,2, \ldots, r$ are failure states ranked according to expected repair times. To arrive at the structure function only perfect functioning or not is of relevance for these components.

Now let $a$ be the module consisting of the components $a_{1}, a_{2}$. We let the set of states be $S_{a}=\{0,1,16\}$, where state 1 is achieved when the state of $a_{2}$ is less than 16 , whereas the state of $a_{1}$ is 16 . The state 16 is achieved when at least the state of $a_{2}$ is 16 . From Figure 2.1 and Table 2.3 one easily arrives at Table 2.4 summing up the states of $a$.

\begin{tabular}{|c|c|}
\hline State & Capacity \\
\hline 0 & 0 \\
\hline 1 & 5 \\
\hline 16 & $20-25$ \\
\hline
\end{tabular}

Table 2.4. The states of module $a$.

The corresponding structure function is given by

$$
a=\phi_{a}\left(a_{1}, a_{2}\right)=I\left(a_{1}=16\right) I\left(a_{2}<16\right)+16 I\left(a_{2}=16\right),
$$

where $I(A)$ equals 1 if $A$ is true and 0 otherwise. For notational convenience, in (2.1) and in the following corresponding equations we denote the state of a module or component by its name.

From Figure 2.1 and Table 2.3 one gets Table 2.5 summing up the states of module $b$.

\begin{tabular}{|c|c|}
\hline State & Capacity \\
\hline 0 & 0 \\
\hline 1 & 4.2 \\
\hline 2 & 8.0 \\
\hline 3 & 10.3 \\
\hline 16 & 16 \\
\hline
\end{tabular}

Table 2.5. The states of module $b$.

The corresponding structure function is given by

$$
\begin{aligned}
b= & \phi_{b}\left(b_{1}, b_{2}, b_{3}, b_{4}, b_{5}, b_{6}, b_{7}, b_{8}\right) \\
= & I\left(\min \left(b_{1}, b_{2}, b_{3}, b_{7}, b_{8}\right)=16\right)\left\{I\left(b_{4}<16\right)\right. \\
& \left.+I\left(b_{4}=16\right)\left[2+I\left(b_{5}=16\right)\left[1+13 I\left(b_{6}=16\right)\right]\right]\right\}
\end{aligned}
$$

Combining the modules $a$ and $b$ into module $a b$, from Tables 2.4 and 2.5 one arrives at Table 2.6 summing up the states of this module. 


\begin{tabular}{|c|c|}
\hline State & Capacity \\
\hline 0 & 0 \\
\hline 1 & 4.2 \\
\hline 2 & 5 \\
\hline 3 & 8.0 \\
\hline 4 & 10.3 \\
\hline 16 & 16 \\
\hline
\end{tabular}

Table 2.6. The states of module $a b$.

The corresponding structure function is given by

$$
\begin{aligned}
a b & =\phi_{a b}(a, b)=I(\min (a, b)>0) \\
& \times\{I(a=1)[1+I(b \geq 2)]+I(a=16)[I(b=1) \\
& +3 I(b=2)+4 I(b=3)+16 I(b=16)]\}
\end{aligned}
$$

Let $c$ be the module consisting of the components $c_{1}$ and $c_{2}$. We let the set of states be $S_{c}=\{0,6,7,16\}$. Here state 6 is achieved when the state of $c_{1}$ is less than 16 , whereas the state of $c_{2}$ is 16 . The state 7 is achieved when it is the other way round, whereas the state 16 is achieved when both the state of $c_{1}$ and $c_{2}$ is 16 . From Figure 2.1 and Table 2.3 one gets Table 2.7 summing up the states of $c$.

\begin{tabular}{|c|c|}
\hline State & Capacity \\
\hline 0 & 0 \\
\hline 6 & 42 \\
\hline 7 & 48 \\
\hline 16 & 90 \\
\hline
\end{tabular}

Table 2.7. The states of module $c$.

The corresponding structure function is given by

$$
\begin{aligned}
c= & \phi_{c}\left(c_{1}, c_{2}\right)=6 I\left(c_{1}<16\right) I\left(c_{2}=16\right)+7 I\left(c_{1}=16\right) I\left(c_{2}<16\right) \\
& +16 I\left(c_{1}=16\right) I\left(c_{2}=16\right)
\end{aligned}
$$

From Figure 2.1 and Table 2.3 one arrives at Table 2.8 summing up the states of module $d$.

\begin{tabular}{|c|c|}
\hline State & Capacity \\
\hline 0 & 0 \\
\hline 3 & 30 \\
\hline 4 & 35 \\
\hline 16 & 50 \\
\hline
\end{tabular}

Table 2.8. The states of module $d$. 
The corresponding structure function is given by

$$
\begin{aligned}
d= & \phi_{d}\left(d_{1}, d_{2}, d_{3}, d_{4}, d_{5}, d_{6}\right)=I\left(\min \left(d_{1}, d_{3}, d_{4}, d_{5}\right)=16\right) \\
& \times\left\{3+I\left(d_{2}<16\right) I\left(d_{6}=16\right)+13 I\left(d_{2}=16\right) I\left(d_{6}=16\right)\right\}
\end{aligned}
$$

Combining the modules $a b, c, d$ and component $e$ into module abcde, from Figure 2.1 and Tables 2.3, 2.6, 2.7, 2.8 one gets Table 2.9 summing up the states of module abcde.

\begin{tabular}{|c|c|}
\hline State & Capacity \\
\hline 0 & 0 \\
\hline 1 & $4.2-19$ \\
\hline 2 & $21.3-27$ \\
\hline 3 & 30 \\
\hline 4 & 35 \\
\hline 5 & $41-42$ \\
\hline 7 & $46-48$ \\
\hline 8 & $50-53$ \\
\hline 16 & $57.2-61$ \\
\hline
\end{tabular}

Table 2.9. The states of module abcde.

The corresponding structure function is given by

$$
\begin{aligned}
& a b c d e=\phi_{a b c d e}(a b, c, d, e) \\
& =I(d=0) I(e=16)+I(d=3)\{I(c=0) \\
& \quad \times[I(e<16) I(a b>0)+I(e=16)(I(a b<4)+2 I(a b \geq 4))] \\
& \quad+I(c>0)[3+2 I(e=16)]\}+I(d=4)\{I(c=0) \\
& \quad \times[I(e<16) I(a b>0)+I(e=16)(I(a b<4)+2 I(a b \geq 4))] \\
& \quad+I(c>0)[4+3 I(e=16)]\}+I(d=16)\{I(c=0) \\
& \quad \times[I(e<16) I(a b>0)+I(e=16)(I(a b<4)+2 I(a b \geq 4))] \\
& \quad+I(c=6)[5+I(e<16)(2 I(1 \leq a b \leq 2)+3 I(a b \geq 3)) \\
& \quad+I(e=16)(3+8 I(a b \geq 1))]+I(c=7)[7 \\
& \quad+I(e<16) I(a b>0)+9 I(e=16)]+I(c=16)[8+8 I(e=16)]\}
\end{aligned}
$$

From Figure 2.1 and Tables 2.1, 2.3 one arrives at Table 2.10 summing up the states of module $f$.

\begin{tabular}{|c|c|}
\hline State & Capacity/Compression \\
\hline 0 & 0 \\
\hline 2 & 11 \\
\hline 4 & 22 \\
\hline 16 & 33 \\
\hline
\end{tabular}

Table 2.10. The states of module $f$. 
The corresponding structure function is given by

$$
\begin{aligned}
f= & \phi_{f}\left(f_{1}, f_{2}\right)=I\left(f_{2}=16\right)\left[2 I\left(2 \leq f_{1} \leq 3\right)\right. \\
& \left.+4 I\left(4 \leq f_{1} \leq 5\right)+16 I\left(14 \leq f_{1} \leq 16\right)\right]
\end{aligned}
$$

Similarly, from Figure 2.1 and Tables 2.2, 2.3 we obtain Table 2.11 summing up the states of modules $h$ and $j$.

\begin{tabular}{|c|c|}
\hline State & Capacity/Compression \\
\hline 0 & 0 \\
\hline 1 & 4.5 \\
\hline 16 & 9 \\
\hline
\end{tabular}

Table 2.11. The states of modules $h$ and $j$.

The corresponding structure functions are given by

$$
\begin{gathered}
h=\phi_{h}\left(h_{1}, h_{2}\right)=I\left(h_{2}=16\right)\left[I\left(2 \leq h_{1} \leq 3\right)+16 I\left(14 \leq h_{1} \leq 16\right)\right] \\
j=\phi_{j}\left(j_{1}, j_{2}\right)=I\left(j_{2}=16\right)\left[I\left(2 \leq j_{1} \leq 3\right)+16 I\left(14 \leq j_{1} \leq 16\right)\right]
\end{gathered}
$$

From Figure 2.1 and Table 2.3 we arrive at Table 2.12 summing up the states of modules $g$ and $i$.

\begin{tabular}{|c|c|}
\hline State & Capacity \\
\hline 0 & 0 \\
\hline 3 & 30 \\
\hline 16 & 59 \\
\hline
\end{tabular}

Table 2.12. The states of modules $g$ and $i$.

The corresponding structure functions are given by

$$
\begin{aligned}
g & =\phi_{g}\left(g_{1}, g_{2}, g_{3}, g_{4}\right)=I\left(\min \left(g_{1}, g_{2}, g_{3}\right)=16\right)\left[3+13 I\left(g_{4}=16\right)\right] \\
i & =\phi_{i}\left(i_{1}, i_{2}\right)=I\left(i_{1}=16\right)\left[3+13 I\left(i_{2}=16\right)\right]
\end{aligned}
$$

At last we are in the position of considering the system as a whole. From Figure 2.1 and Tables 2.9, 2.10, 2.11, 2.12 we obtain Table 2.13 summing up the system states. In this table mainly the amount of gas compressed by module $j$, being closest to Emden, is taken into account, but only when the amount of transported gas is at least $50 \mathrm{MSm}^{3} / d$. In addition a "+" in the compression column indicates the advantageous situation where all compressor modules $f, h$ and $j$ are in state 16 . Of course this simplifying approach is not necessary, but as is seen, even this approach leads to a rather complex system structure function. 


\begin{tabular}{|c|c|c|}
\hline State & Capacity & Compression \\
\hline 0 & 0 & - \\
\hline 1 & $4.2-19$ & - \\
\hline 2 & $21.3-27$ & - \\
\hline 3 & 30 & - \\
\hline 4 & 35 & - \\
\hline 5 & $41-42$ & - \\
\hline 6 & 45 & - \\
\hline 7 & $46-48$ & - \\
\hline 8 & 50 & 0 \\
\hline 9 & $50-53$ & 4.5 \\
\hline 10 & $50-53$ & 9 \\
\hline 11 & $50-53$ & $9^{+}$ \\
\hline 12 & 54.5 & 4.5 \\
\hline 13 & 54.5 & 9 \\
\hline 14 & 56 & 9 \\
\hline 15 & $57.2-59$ & 9 \\
\hline 16 & $57.2-59$ & $9^{+}$ \\
\hline
\end{tabular}

Table 2.13. System states.

Some careful thinking leads to the following system structure function

$$
\begin{aligned}
& \phi(a b c d e, f, g, h, i, j, k) \\
& =I(k=16) I(\min (a b c d e, g, i)>0) \\
& \{I(a b c d e \leq 3) a b c d e+I(a b c d e=4)[3+I(g=16) I(i=16)] \\
& +I(a b c d e=5)[3+2 I(g=16) I(i=16)] \\
& +I(a b c d e=7)[3+I(g=16) I(i=16)(3 I(f=0)+4 I(f>0))] \\
& +I(a b c d e=8)[3+I(g=16) I(i=16)\{3 I(f=0) \\
& +I(2 \leq f \leq 4)(5+I(j=1)+2 I(j=16)) \\
& +I(f=16)[I(h \leq 1)(5+I(j=1)+2 I(j=16)) \\
& +I(h=16)(5+I(j=1)+3 I(j=16))]\}] \\
& +I(a b c d e=16)[3+I(g=16) I(i=16)\{3 I(f=0) \\
& +I(f=2)[I(h=0)(5+I(j=1)+2 I(j=16)) \\
& +I(h=1)(5+4 I(j=1)+5 I(j=16)) \\
& +I(h=16)(5+4 I(j=1)+6 I(j=16))] \\
& +I(f=4)[I(h=0)(5+I(j=1)+2 I(j=16)) \\
& +I(h=1)(5+4 I(j=1)+5 I(j=16)) \\
& +I(h=16)(5+4 I(j=1)+7 I(j=16)] \\
& +I(f=16)[I(h=0)(5+I(j=1)+2 I(j=16)) \\
& +I(h=1)(5+4 I(j=1)+5 I(j=16)) \\
& +I(h=16)(5+4 I(j=1)+8 I(j=16))]\}]\}
\end{aligned}
$$




\section{Availabilities and unavailabilities of the compo- nents}

As mentioned at the end of Section 1, bounds for the availabilities and unavailabilities in a fixed time interval for an MMS are based on corresponding information on the multistate components. Hence, with obvious definitions, denote the availability and unavailability to level $j$ in the time interval $I$ for the $i$ th component of an MMS by $p_{i}^{j(I)}$ and $q_{i}^{j(I)}$ respectively, $i=1, \ldots, n ; j \in\{1, \ldots, M\}$. In this section we establish these quantities for the components of the offshore gas pipeline network.

Assume that the performance processes of the components are Markovian and introduce in the general case

$$
\begin{aligned}
S_{i, j}^{0} & =S_{i} \cap\{0,1, \ldots, j-1\} \\
S_{i, j}^{1} & =S_{i} \cap\{j, \ldots, M\} \\
p_{i}^{(k, \ell)}\left(t_{1}, t_{2}\right) & =P\left(X_{i}\left(t_{2}\right)=\ell \mid X_{i}\left(t_{1}\right)=k\right) .
\end{aligned}
$$

Furthermore, denote the corresponding transition probabilities when $E$ is a set of absorbing states by $p_{i}^{(k, \ell) E}\left(t_{1}, t_{2}\right)$. Finally, assume that at time $t=0$ all components are in the perfect functioning state $M$; i.e. $\boldsymbol{X}(0)=\boldsymbol{M}$. Then for $I=\left[t_{1}, t_{2}\right]$

$$
\begin{aligned}
& p_{i}^{j(I)}=\sum_{k \in S_{i, j}^{1}} p_{i}^{(M, k)}\left(0, t_{1}\right)\left[1-\sum_{\ell \in S_{i, j}^{0}} p_{i}^{(k, \ell) S_{i j}^{0}}\left(t_{1}, t_{2}\right)\right] \\
& q_{i}^{j(I)}=\sum_{k \in S_{i, j}^{0}} p_{i}^{(M, k)}\left(0, t_{1}\right)\left[1-\sum_{\ell \in S_{i, j}^{1}} p_{i}^{(k, \ell) S_{i j}^{1}}\left(t_{1}, t_{2}\right)\right] .
\end{aligned}
$$

Note that we get $q_{i}^{j(I)}$ from $p_{i}^{j(I)}$ by replacing $S_{i j}^{1}$ by the 'dual' set $S_{i j}^{0}$.

Now let

$$
\mu_{i}^{(k, \ell)}(s)=\lim _{h \rightarrow 0} p_{i}^{(k, \ell)}(s, s+h) / h \quad k \neq \ell,
$$

be the transition intensities of $\left\{X_{i}(t), t \in[0, \infty)\right\}$. For simplicity we assume that the performance processes of the components are time-homogeneous, i.e.

$$
\begin{aligned}
& p_{i}^{(k, \ell)}\left(t_{1}, t_{2}\right)=p_{i}^{(k, \ell)}\left(t_{2}-t_{1}\right) \\
& \mu_{i}^{(k, \ell)}(s)=\mu_{i}^{(k, \ell)} \quad \text { for all } s \in[0, \infty), k \neq \ell .
\end{aligned}
$$

Consequently, all that is needed to arrive at expressions for $p_{i}^{j(I)}$ and $q_{i}^{j(I)}$, and hence bounds for $h_{\phi}^{j(I)}$ and $g_{\phi}^{j(I)}$, are these time-independent transition intensities.

Now introduce the matrices

$$
\boldsymbol{P}_{i}(t)=\left\{p_{i}^{(k, \ell)}(t)\right\}_{k \in S_{i}, \ell \in S_{i}}
$$


Since the set $S_{i}$ is finite, the performance processes of the components are conservative, implying that the corresponding intensity matrices are on the form $(i=$ $1, \ldots, n)$

$$
\boldsymbol{A}_{i}=\left[\begin{array}{ccc}
-\sum_{k=1}^{M} \mu_{0 k} & \cdots \cdots \cdots & \mu_{0 M} \\
\vdots & & \vdots \\
\vdots & & \vdots \\
\mu_{M 0} & \cdots \cdots \cdots & -\sum_{k=0}^{M-1} \mu_{M k}
\end{array}\right]
$$

Denoting $\left|S_{i}\right|$ the cardinality of the set $S_{i}, \boldsymbol{A}_{i}$ is an $\left|S_{i}\right| \times\left|S_{i}\right|$ matrix.

By applying standard theory for finite-state continuous-time Markov processes, see Karlin and Taylor [5], we have

$$
\boldsymbol{P}_{i}(t)=\exp \left(\boldsymbol{A}_{i}(t)\right)=\boldsymbol{I}+\sum_{n=1}^{\infty} \frac{\boldsymbol{A}_{i}^{n} t^{n}}{n !},
$$

where $\boldsymbol{I}$ is the identity matrix and the initial condition is $\boldsymbol{P}_{i}(0)=\boldsymbol{I}$.

The availabilities and unavailabilities of the 32 components of the offshore gas pipeline system are determined by the computer package MUSTAFA (MUltiSTAte Fault tree Analysis) developed by Høgåsen [4]. The only input needed are the intensity matrices of the components, implicitly giving the corresponding sets of states, along with the time interval $I$.

The intensity matrices of $a_{1}$ and the compressor component $f_{1}$ are given below. For the interested reader, aiming for instance at checking our results by a simulation study, the remaining intensity matrices are given in the appendix.

$$
\boldsymbol{A}_{a_{1}}=\left[\begin{array}{ccccc}
-4 & 0 & 0 & 0 & 4 \\
0 & -8.3721 & 0 & 0 & 8.3721 \\
0 & 0 & -9 & 0 & 9 \\
0 & 0 & 0 & -22.25 & 22.25 \\
0.00003 & 0.0019 & 0.028 & 0.0061 & -0.03603
\end{array}\right]
$$

Note that $S_{a_{1}}=\{0,1,2,3,16\}$, and that the failure states are ranked according to inverse repair rates or, equivalently, the expected repair times.

$$
A_{f_{1}}=\left[\begin{array}{ccccccccc}
-1783.35 & 0 & 1711.35 & 72 & 0 & 0 & 0 & 0 & 0 \\
0 & -2281.8 & 0 & 2281.8 & 0 & 0 & 0 & 0 & 0 \\
9.6 & 0 & -1222.5 & 0 & 1140.9 & 72 & 0 & 0 & 0 \\
0 & 9.6 & 0 & -1720.95 & 0 & 1711.35 & 0 & 0 & 0 \\
0 & 0 & 19.2 & 0 & -661.7 & 0 & 570.45 & 72 & 0 \\
0 & 0 & 0 & 19.2 & 0 & -1160.1 & 0 & 1140.9 & 0 \\
0 & 0 & 0 & 0 & 28.8 & 0 & -100.8 & 0 & 72 \\
0 & 0 & 0 & 0 & 0 & 28.8 & 0 & -599.25 & 570.45 \\
0 & 0 & 0 & 0 & 0 & 0 & 20 & 38.4 & -58.4
\end{array}\right]
$$

Note that the maintenance completion rate of 72 completions per year is smaller than the repair rate of a single compressor of 570.45 repairs per year as assumed when ranking the states of $f_{1}$ in Table 2.1, and that there are four repairmen. Note also that a compressor is only sent for maintenance when all four compressors are operative, and that in this case the fourth compressor is in hot standby. 


\section{Bounds for the availabilities and unavailabilities for the offshore gas pipeline network}

As an example of the bounds for $h_{\phi}^{j(I)}$ and $g_{\phi}^{j(I)}$ given in Funnemark and Natvig [3] and Natvig [9], without any assumption according to Natvig [11] that each of the performance processes of the components is associated in $I$, we give the following theorem by first introducing the $n \times M$ matrices

$$
\boldsymbol{P}_{\phi}^{(I)}=\left\{p_{i}^{j(I)}\right\}_{\substack{i=1, \ldots, n \\ j=1, \ldots, M}} \quad \boldsymbol{Q}_{\phi}^{(I)}=\left\{q_{i}^{j(I)}\right\}_{\substack{i=1, \ldots, n \\ j=1, \ldots, M}} .
$$

Theorem 4.1 Let $(C, \phi)$ be an MMS with the marginal performance processes of its components being independent in $I$. Furthermore, for $j \in\{1, \ldots, M\}$ let $\boldsymbol{y}_{k}^{j}=$ $\left(y_{1 k}^{j}, \ldots, y_{n k}^{j}\right), k=1, \ldots, n^{j}\left(\boldsymbol{z}_{k}^{j}=\left(z_{1 k}^{j}, \ldots, z_{n k}^{j}\right), k=1, \ldots, m^{j}\right)$ be its minimal path (cut) vectors to level $j$. Define

$$
\begin{aligned}
\ell_{\phi}^{j^{\prime}}\left(\boldsymbol{P}_{\phi}^{(I)}\right) & =\max _{1 \leq k \leq n^{j}} \prod_{i=1}^{n} p_{i}^{y_{i k}^{j}(I)} \quad \bar{\ell}_{\phi}^{j^{\prime}}\left(\boldsymbol{Q}_{\phi}^{(I)}\right)=\max _{1 \leq k \leq m^{j}} \prod_{i=1}^{n} q_{i}^{z_{i k}^{j}+1(I)} \\
\ell_{\phi}^{j^{*}}\left(\boldsymbol{P}_{\phi}^{(I)}\right) & =\prod_{k=1}^{m^{j}} \coprod_{i=1}^{n} p_{i}^{z_{i k}^{j}+1(I)} \quad \bar{\ell}_{\phi}^{j^{*}}\left(\boldsymbol{Q}_{\phi}^{(I)}\right)=\prod_{k=1}^{n^{j}} \coprod_{i=1}^{n} q_{i}^{y_{i k}^{j}(I)} \\
B_{\phi}^{j}\left(\boldsymbol{P}_{\phi}^{(I)}\right) & =\max _{j \leq k \leq M}\left\{\max \left[\ell_{\phi}^{k^{\prime}}\left(\boldsymbol{P}_{\phi}^{(I)}\right), \ell_{\phi}^{k^{*}}\left(\boldsymbol{P}_{\phi}^{(I)}\right)\right]\right\} \\
\bar{B}_{\phi}^{j}\left(\boldsymbol{Q}_{\phi}^{(I)}\right) & =\max _{1 \leq k \leq j}\left\{\max \left[\ell_{\phi}^{k^{\prime}}\left(\boldsymbol{Q}_{\phi}^{(I)}\right), \bar{\ell}_{\phi}^{k^{*}}\left(\boldsymbol{Q}_{\phi}^{(I)}\right)\right]\right\}
\end{aligned}
$$

Then

$$
\begin{aligned}
& B_{\phi}^{j}\left(\boldsymbol{P}_{\phi}^{(I)}\right) \leq h_{\phi}^{j(I)} \leq \inf _{t \in I}\left[1-\bar{B}_{\phi}^{j}\left(\boldsymbol{Q}_{\phi}^{([t, t])}\right)\right] \leq 1-\bar{B}_{\phi}^{j}\left(\boldsymbol{Q}_{\phi}^{(I)}\right) \\
& \bar{B}_{\phi}^{j}\left(\boldsymbol{Q}_{\phi}^{(I)}\right) \leq g_{\phi}^{j(I)} \leq \inf _{t \in I}\left[1-B_{\phi}^{j}\left(\boldsymbol{P}_{\phi}^{([t, t])}\right)\right] \leq 1-B_{\phi}^{j}\left(\boldsymbol{P}_{\phi}^{(I)}\right) .
\end{aligned}
$$

Here $\coprod_{i=1}^{n} a_{i} \stackrel{\text { def }}{=} 1-\prod_{i=1}^{n}\left(1-a_{i}\right)$. By specializing $M=1$ and $I=[t, t]$ the bounds reduce to the familiar ones from binary theory as given in Barlow and Proschan [1].

By using the computer package MUSTAFA, which finds the minimal path and cut vectors to all levels from equations (2.1)-(2.12) and then applies the best bounds of Theorem 4.1, we arrive at the bounds shown in Table 4.1, which are not based on a modular decomposition. Note that for the case $I=[0.5,0.5]$ we know from Section 1 that $g_{\phi}^{j[0.5,0.5]}=1-h_{\phi}^{j[0.5,0.5]}$. Hence, the bounds for $g_{\phi}^{j[0.5,0.5]}$ follow immediately from the ones of $h_{\phi}^{j[0.5,0.5]}$. Furthermore, note that the upper bounds both for $h_{\phi}^{j[0.5,0.6]}$ and $h_{\phi}^{j[0.5,0.5]}$, and also for $g_{\phi}^{j[0.5,0.6]}$ and $g_{\phi}^{j[0.5,0.5]}$, are almost completely identical. This is due to the fact that the upper bounds for a fixed time point $t$ in Theorem 4.1 do 
not depend on $t$ for $t \geq 0.5$. Hence, stationarity is reached after half a year. We see that all bounds are very informative for $I=[0.5,0.5]$ and for the unavailabilities also for $I=[0.5,0.6]$, corresponding to an interval length of 36 days. They are less informative for the availabilities for $I=[0.5,0.6]$. Note, however, that from the theory we know that all lower bounds are far better than the corresponding upper bounds especially for long intervals. Also, to be on the conservative side, the lower bounds for the availabilities are the most interesting.

It should be remarked that none of the intervals in Table 4.1 reduce to a single exact value. This follows from the theory in Natvig [11] since there are more than one single minimal path and minimal cut vector to all levels. Exact values are obtained in Mørch [6] for some simpler networks. 


\begin{tabular}{|c|c|c|c|c|c|}
\hline \multirow[t]{2}{*}{$j$} & \multirow[t]{2}{*}{$I$} & \multicolumn{2}{|c|}{ bounds for $h_{\phi}^{j(I)}$} & \multicolumn{2}{|c|}{ bounds for $g_{\phi}^{j(I)}$} \\
\hline & & lower & upper & lower & upper \\
\hline \multirow{2}{*}{16} & {$[0.5,0.6]$} & 0.0897 & 0.9600 & 0.0120 & 0.0836 \\
\hline & {$[0.5,0.5]$} & 0.9164 & 0.9601 & 0.0399 & 0.0836 \\
\hline \multirow{2}{*}{15} & {$[0.5,0.6]$} & 0.1672 & 0.9600 & 0.0120 & 0.0738 \\
\hline & {$[0.5,0.5]$} & 0.9262 & 0.9601 & 0.0399 & 0.0738 \\
\hline \multirow{2}{*}{14} & {$[0.5,0.6]$} & 0.1706 & 0.9600 & 0.0120 & 0.0737 \\
\hline & {$[0.5,0.5]$} & 0.9263 & 0.9601 & 0.0399 & 0.0737 \\
\hline \multirow{2}{*}{13} & {$[0.5,0.6]$} & 0.2422 & 0.9600 & 0.0120 & 0.0686 \\
\hline & {$[0.5,0.5]$} & 0.9314 & 0.9601 & 0.0399 & 0.0686 \\
\hline \multirow{2}{*}{12} & {$[0.5,0.6]$} & 0.3439 & 0.9600 & 0.0120 & 0.0635 \\
\hline & {$[0.5,0.5]$} & 0.9365 & 0.9601 & 0.0399 & 0.0635 \\
\hline \multirow{2}{*}{11} & {$[0.5,0.6]$} & 0.3439 & 0.9968 & 0.0011 & 0.0254 \\
\hline & {$[0.5,0.5]$} & 0.9746 & 0.9968 & 0.0032 & 0.0254 \\
\hline \multirow{2}{*}{10} & {$[0.5,0.6]$} & 0.3904 & 0.9968 & 0.0011 & 0.0230 \\
\hline & {$[0.5,0.5]$} & 0.9770 & 0.9968 & 0.0032 & 0.0230 \\
\hline \multirow{2}{*}{9} & {$[0.5,0.6]$} & 0.4113 & 0.9968 & 0.0011 & 0.0228 \\
\hline & {$[0.5,0.5]$} & 0.9772 & 0.9968 & 0.0032 & 0.0228 \\
\hline \multirow{2}{*}{8} & {$[0.5,0.6]$} & 0.4492 & 0.9968 & 0.0011 & 0.0211 \\
\hline & {$[0.5,0.5]$} & 0.9789 & 0.9968 & 0.0032 & 0.0211 \\
\hline \multirow{2}{*}{7} & {$[0.5,0.6]$} & 0.4566 & 0.9968 & 0.0011 & 0.0207 \\
\hline & {$[0.5,0.5]$} & 0.9793 & 0.9968 & 0.0032 & 0.0207 \\
\hline \multirow{2}{*}{6} & {$[0.5,0.6]$} & 0.4720 & 0.9968 & 0.0011 & 0.0199 \\
\hline & {$[0.5,0.5]$} & 0.9801 & 0.9968 & 0.0032 & 0.0199 \\
\hline \multirow{2}{*}{5} & {$[0.5,0.6]$} & 0.4802 & 0.9968 & 0.0011 & 0.0195 \\
\hline & {$[0.5,0.5]$} & 0.9805 & 0.9968 & 0.0032 & 0.0195 \\
\hline \multirow{2}{*}{4} & {$[0.5,0.6]$} & 0.4810 & 0.9968 & 0.0011 & 0.0195 \\
\hline & {$[0.5,0.5]$} & 0.9805 & 0.9968 & 0.0032 & 0.0195 \\
\hline \multirow{2}{*}{3} & {$[0.5,0.6]$} & 0.4952 & 0.9968 & 0.0011 & 0.0189 \\
\hline & {$[0.5,0.5]$} & 0.9811 & 0.9968 & 0.0032 & 0.0189 \\
\hline \multirow{2}{*}{2} & {$[0.5,0.6]$} & 0.4952 & 0.9968 & 0.0011 & 0.0189 \\
\hline & {$[0.5,0.5]$} & 0.9811 & 0.9968 & 0.0032 & 0.0189 \\
\hline \multirow{2}{*}{1} & {$[0.5,0.6]$} & 0.7354 & 0.9975 & 0.0011 & 0.0112 \\
\hline & {$[0.5,0.5]$} & 0.9888 & 0.9975 & 0.0025 & 0.0112 \\
\hline
\end{tabular}

Table 4.1. Bounds for $h_{\phi}^{j(I)}$ and $g_{\phi}^{j(I)}$ not based on a modular decomposition.

The computer package MUSTAFA can also find a modular decomposition of a system. Then bounds for the availabilities and unavailabilities for the modules can be established from the availabilities and unavailabilities of the components. Furthermore, bounds for the availabilities and unavailabilities of the system can be established from these bounds for the availabilities and unavailabilities for the modules. Using this approach we arrive at the best bounds shown in Table 4.2. 
First note that the comments made on Table 4.1 are still valid for Table 4.2. Secondly, note that the bounds for a fixed time point $t=0.5$ are more informative in Table 4.2 than in Table 4.1. This is in accordance with the theory of Funnemark \& Natvig [3], Natvig [9] and Natvig [11]. The same is also true to some extent for the interval $I=[0.5,0.6]$, but this is not true in general. For instance, Table 4.1 gives $0.3904 \leq h_{\phi}^{10[0.5,0.6]} \leq 0.9968$, whereas Table 4.2 gives $0.3439 \leq h_{\phi}^{10[0.5,0.6]} \leq 0.9828$.

\begin{tabular}{|c|c|c|c|c|c|}
\hline \multirow[t]{2}{*}{$j$} & \multirow[t]{2}{*}{$I$} & \multicolumn{2}{|c|}{ bounds for $h_{\phi}^{j(I)}$} & \multicolumn{2}{|c|}{ bounds for $g_{\phi}^{j(I)}$} \\
\hline & & lower & upper & lower & upper \\
\hline \multirow{2}{*}{16} & {$[0.5,0.6]$} & 0.0897 & 0.9246 & 0.0148 & 0.0836 \\
\hline & {$[0.5,0.5]$} & 0.9164 & 0.9247 & 0.0753 & 0.0836 \\
\hline \multirow{2}{*}{15} & {$[0.5,0.6]$} & 0.1672 & 0.9344 & 0.0148 & 0.0738 \\
\hline & {$[0.5,0.5]$} & 0.9262 & 0.9345 & 0.0655 & 0.0738 \\
\hline \multirow{2}{*}{14} & {$[0.5,0.6]$} & 0.1706 & $\overline{0.9346}$ & 0.0148 & 0.0737 \\
\hline & {$[0.5,0.5]$} & 0.9263 & 0.9347 & 0.0653 & 0.0737 \\
\hline \multirow{2}{*}{13} & {$[0.5,0.6]$} & 0.2422 & 0.9397 & 0.0148 & 0.0686 \\
\hline & {$[0.5,0.5]$} & 0.9314 & 0.9398 & 0.0602 & 0.0686 \\
\hline \multirow{2}{*}{12} & {$[0.5,0.6]$} & 0.3439 & 0.9449 & 0.0148 & 0.0635 \\
\hline & {$[0.5,0.5]$} & 0.9365 & 0.9450 & 0.0550 & 0.0635 \\
\hline \multirow{2}{*}{11} & {$[0.5,0.6]$} & 0.3439 & 0.9828 & 0.0034 & 0.0257 \\
\hline & {$[0.5,0.5]$} & 0.9743 & 0.9829 & 0.0171 & 0.0257 \\
\hline \multirow{2}{*}{10} & {$[0.5,0.6]$} & 0.3439 & 0.9828 & 0.0034 & 0.0232 \\
\hline & {$[0.5,0.5]$} & 0.9768 & 0.9829 & 0.0171 & 0.0232 \\
\hline \multirow{2}{*}{9} & {$[0.5,0.6]$} & 0.4113 & 0.9828 & 0.0034 & 0.0228 \\
\hline & {$[0.5,0.5]$} & 0.9772 & 0.9829 & 0.0171 & 0.0228 \\
\hline \multirow{2}{*}{8} & {$[0.5,0.6]$} & 0.4492 & 0.9845 & 0.0033 & 0.0211 \\
\hline & {$[0.5,0.5]$} & 0.9789 & 0.9846 & 0.0154 & 0.0211 \\
\hline \multirow{2}{*}{7} & {$[0.5,0.6]$} & 0.4566 & 0.9845 & 0.0033 & 0.0207 \\
\hline & {$[0.5,0.5]$} & 0.9793 & 0.9846 & 0.0154 & 0.0207 \\
\hline \multirow{2}{*}{6} & {$[0.5,0.6]$} & 0.4720 & 0.9853 & 0.0033 & 0.0199 \\
\hline & {$[0.5,0.5]$} & 0.9801 & 0.9854 & 0.0146 & 0.0199 \\
\hline \multirow{2}{*}{5} & {$[0.5,0.6]$} & 0.4802 & 0.9853 & 0.0033 & 0.0195 \\
\hline & {$[0.5,0.5]$} & 0.9805 & 0.9854 & 0.0146 & 0.0195 \\
\hline \multirow{2}{*}{4} & {$[0.5,0.6]$} & 0.4810 & 0.9853 & 0.0033 & 0.0195 \\
\hline & {$[0.5,0.5]$} & 0.9805 & 0.9854 & 0.0146 & 0.0195 \\
\hline \multirow{2}{*}{3} & {$[0.5,0.6]$} & 0.4952 & 0.9859 & 0.0033 & 0.0189 \\
\hline & {$[0.5,0.5]$} & 0.9811 & 0.9860 & 0.0140 & 0.0189 \\
\hline \multirow{2}{*}{2} & {$[0.5,0.6]$} & 0.4952 & 0.9859 & 0.0033 & 0.0189 \\
\hline & {$[0.5,0.5]$} & 0.9811 & 0.9860 & 0.0140 & 0.0189 \\
\hline \multirow{2}{*}{1} & {$[0.5,0.6]$} & 0.7354 & 0.9889 & 0.0027 & 0.0112 \\
\hline & {$[0.5,0.5]$} & 0.9888 & 0.9890 & 0.0110 & 0.0112 \\
\hline
\end{tabular}

Table 4.2. Bounds for $h_{\phi}^{j(I)}$ and $g_{\phi}^{j(I)}$ based on a modular decomposition found by MUSTAFA. 
Finally, it should be concluded that having the computer package MUSTAFA more complex systems than the one treated here can be attacked.

\section{Acknowledgements}

We are very thankful to senior discipline leader Morten Sørum for providing information and knowledge on the offshore gas pipeline network considered, to dr.scient Gutorm Høgåsen for patience in answering questions on the computer package MUSTAFA, and to three referees for a careful reading of the manuscript and constructive comments.

\section{Appendix}

\section{Remaining intensity matrices of the components}

$$
\begin{aligned}
& A_{a_{2}}=\left[\begin{array}{ccccc}
-4 & 0 & 0 & 0 & 4 \\
0 & -8.3721 & 0 & 0 & 8.3721 \\
0 & 0 & -9 & 0 & 9 \\
0 & 0 & 0 & -22.5 & 22.5 \\
0.00006 & 0.01565 & 0.021 & 0.01736 & -0.05406
\end{array}\right] \\
& A_{b_{1}}=\left[\begin{array}{cccccc}
-1.8 & 0 & 0 & 0 & 0 & 1.8 \\
0 & -6.207 & 0 & 0 & 0 & 6.207 \\
0 & 0 & -7.3469 & 0 & 0 & 7.3469 \\
0 & 0 & 0 & -8.3721 & 0 & 8.3721 \\
0 & 0 & 0 & 0 & -9 & 9 \\
0.000066 & 0.000072 & 0.0023 & 0.0082 & 0.02 & -0.03064
\end{array}\right] \\
& A_{b_{2}}=\left[\begin{array}{ccccc}
-22.5 & 0 & 0 & 0 & 22.5 \\
0 & -51.4286 & 0 & 0 & 51.4286 \\
0 & 0 & -444.4444 & 0 & 444.4444 \\
0 & 0 & 0 & -720 & 720 \\
0.109 & 0.0008 & 0.31 & 0.03 & -0.4498
\end{array}\right] \\
& A_{b_{3}}=\left[\begin{array}{cccccc}
-3.6735 & 0 & 0 & 0 & 0 & 3.6735 \\
0 & -51.4286 & 0 & 0 & 0 & 51.4286 \\
0 & 0 & -120 & 0 & 0 & 120 \\
0 & 0 & 0 & -180 & 0 & 180 \\
0 & 0 & 0 & 0 & -360 & 360 \\
0.00099 & 0.00017 & 0.0017 & 0.05 & 0.44 & -0.49286
\end{array}\right] \\
& A_{b_{4}}=\left[\begin{array}{cc}
-120 & 120 \\
0.08 & -0.08
\end{array}\right] \\
& A_{b_{5}}=\left[\begin{array}{ccccc}
-18 & 0 & 0 & 0 & 18 \\
0 & -51.4286 & 0 & 0 & 51.4286 \\
0 & 0 & -180 & 0 & 180 \\
0 & 0 & 0 & -360 & 360 \\
0.0085 & 0.00089 & 1.6 & 1.06 & -2.66939
\end{array}\right] \\
& A_{b_{6}}=\left[\begin{array}{cccc}
-180 & 0 & 0 & 180 \\
0 & -211.7647 & 0 & 211.7647 \\
0 & 0 & -360 & 360 \\
1.8 & 1.3 & 0.08 & -3.18
\end{array}\right] \\
& A_{b_{7}}=\left[\begin{array}{cccccc}
-7.3469 & 0 & 0 & 0 & 0 & 7.3469 \\
0 & -8.3721 & 0 & 0 & 0 & 8.3721 \\
0 & 0 & -22.5 & 0 & 0 & 22.5 \\
0 & 0 & 0 & -51.4286 & 0 & 51.4286 \\
0 & 0 & 0 & 0 & -444.4444 & 444.4444 \\
0.00154 & 0.00554 & 0.09402 & 0.0008 & 0.176 & -0.2779
\end{array}\right] \\
& A_{b_{8}}=A_{c_{1}}=\left[\begin{array}{cc}
-22.5 & 22.5 \\
0.009 & -0.009
\end{array}\right] \\
& A_{c_{2}}=\left[\begin{array}{cccc}
-4 & 0 & 0 & 4 \\
0 & -8.3721 & 0 & 8.3721 \\
0 & 0 & -22.5 & 22.5 \\
0.00003 & 0.00636 & 0.01704 & -0.02343
\end{array}\right]
\end{aligned}
$$




$$
\begin{aligned}
& A_{d_{1}}=\left[\begin{array}{cccccccc}
-1 & 0 & 0 & 0 & 0 & 0 & 0 & 1 \\
0 & -1.2 & 0 & 0 & 0 & 0 & 0 & 1.2 \\
0 & 0 & -6 & 0 & 0 & 0 & 0 & 6 \\
0 & 0 & 0 & -12 & 0 & 0 & 0 & 12 \\
0 & 0 & 0 & 0 & -22.5 & 0 & 0 & 22.5 \\
0 & 0 & 0 & 0 & 0 & -360 & 0 & 360 \\
0 & 0 & 0 & 0 & 0 & 0 & -900 & 900 \\
0.00022 & 0.0004 & 0.00031 & 0.0017 & 0.009 & 0.14 & 1.8 & -1.95163
\end{array}\right] \\
& A_{d_{2}}=\left[\begin{array}{cc}
-444.4444 & 444.4444 \\
0.175 & -0.175
\end{array}\right] \\
& A_{d_{3}}=\left[\begin{array}{ccc}
-8.3721 & 0 & 8.3721 \\
0 & -22.5 & 22.5 \\
0.01276 & 0.00004 & -0.0128
\end{array}\right] \\
& A_{d_{4}}=\left[\begin{array}{cccccc}
-22.5 & 0 & 0 & 0 & 0 & 22.5 \\
0 & -360 & 0 & 0 & 0 & 360 \\
0 & 0 & -444.4444 & 0 & 0 & 444.4444 \\
0 & 0 & 0 & -720 & 0 & 720 \\
0 & 0 & 0 & 0 & -900 & 900 \\
0.0026 & 0.0017 & 0.38 & 0.06 & 1.8 & -2.2443
\end{array}\right] \\
& A_{d_{5}}=\left[\begin{array}{ccccccc}
-1 & 0 & 0 & 0 & 0 & 0 & 1 \\
0 & -1.2 & 0 & 0 & 0 & 0 & 1.2 \\
0 & 0 & -2 & 0 & 0 & 0 & 2 \\
0 & 0 & 0 & -3 & 0 & 0 & 3 \\
0 & 0 & 0 & 0 & -6 & 0 & 6 \\
0 & 0 & 0 & 0 & 0 & -12 & 12 \\
0.00022 & 0.00022 & 0.00007 & 0.00041 & 0.00036 & 0.00111 & -0.00239
\end{array}\right] \\
& A_{d_{6}}=\left[\begin{array}{ccc}
-444.4444 & 0 & 444.4444 \\
0 & -720 & 720 \\
0.165 & 0.02 & -0.185
\end{array}\right] \\
& A_{e}=\left[\begin{array}{cc}
-12 & 12 \\
0.5 & -0.5
\end{array}\right] \\
& A_{f_{2}}=\left[\begin{array}{cccc}
-12 & 0 & 0 & 12 \\
0 & -360 & 0 & 360 \\
0 & 0 & -444.4444 & 444.4444 \\
0.0014 & 0.008 & 0.311 & -0.3204
\end{array}\right] \\
& A_{g_{1}}=\left[\begin{array}{ccccccccc}
-1 & 0 & 0 & 0 & 0 & 0 & 0 & 0 & 1 \\
0 & -1.2 & 0 & 0 & 0 & 0 & 0 & 0 & 1.2 \\
0 & 0 & -4 & 0 & 0 & 0 & 0 & 0 & 4 \\
0 & 0 & 0 & -6 & 0 & 0 & 0 & 0 & 6 \\
0 & 0 & 0 & 0 & -12 & 0 & 0 & 0 & 12 \\
0 & 0 & 0 & 0 & 0 & -22.5 & 0 & 0 & 22.5 \\
0 & 0 & 0 & 0 & 0 & 0 & -444.4444 & 0 & 444.4444 \\
0 & 0 & 0 & 0 & 0 & 0 & 0 & -720 & 720 \\
0.00066 & 0.00026 & 0.0001 & 0.00062 & 0.003 & 0.008 & 0.301 & 0.02 & -0.33364
\end{array}\right] \\
& A_{g_{2}}=\left[\begin{array}{cccccc}
-1 & 0 & 0 & 0 & 0 & 1 \\
0 & -6 & 0 & 0 & 0 & 6 \\
0 & 0 & -12 & 0 & 0 & 12 \\
0 & 0 & 0 & -360 & 0 & 360 \\
0 & 0 & 0 & 0 & -900 & 900 \\
0.00007 & 0.0001 & 0.0001 & 0.14 & 1.8 & -1.94027
\end{array}\right] \\
& A_{g_{3}}=A_{i_{1}}=\left[\begin{array}{cccccc}
-2 & 0 & 0 & 0 & 0 & 2 \\
0 & -6 & 0 & 0 & 0 & 6 \\
0 & 0 & -8.3721 & 0 & 0 & 8.3721 \\
0 & 0 & 0 & -22.5 & 0 & 22.5 \\
0 & 0 & 0 & 0 & -360 & 360 \\
0.0004 & 0.0003 & 0.00506 & 0.018 & 0.14 & -0.16376
\end{array}\right] \\
& A_{g_{4}}=A_{i_{2}}=\left[\begin{array}{cccc}
-360 & 0 & 0 & 360 \\
0 & -444.4444 & 0 & 444.4444 \\
0 & 0 & -720 & 720 \\
0.008 & 0.107 & 0.02 & -0.135
\end{array}\right] \\
& A_{h_{1}}=A_{j_{1}}=\left[\begin{array}{ccccccc}
-1212.9 & 0 & 1140.9 & 72 & 0 & 0 & 0 \\
0 & -1711.35 & 0 & 1711.35 & 0 & 0 & 0 \\
9.6 & 0 & -652.05 & 0 & 570.45 & 72 & 0 \\
0 & 9.6 & 0 & -1150.5 & 0 & 1140.9 & 0 \\
0 & 0 & 19.2 & 0 & -91.2 & 0 & 72 \\
0 & 0 & 0 & 19.2 & 0 & -589.65 & 570.45 \\
0 & 0 & 0 & 0 & 15 & 28.8 & -43.8
\end{array}\right]
\end{aligned}
$$




$$
\begin{aligned}
A_{h_{2}} & =A_{j_{2}}=\left[\begin{array}{cccccc}
-12 & 0 & 0 & 0 & 0 & 12 \\
0 & -360 & 0 & 0 & 0 & 360 \\
0 & 0 & -444.4444 & 0 & 0 & 444.4444 \\
0 & 0 & 0 & -450 & 0 & 450 \\
0 & 0 & 0 & 0 & -720 & 720 \\
0.0014 & 0.104 & 0.197 & 0.18 & 0.33 & -0.8124
\end{array}\right] \\
A_{k} & =\left[\begin{array}{cccc}
-8.3721 & 0 & 0 & 8.3721 \\
0 & -22.5 & 0 & 22.5 \\
0 & 0 & -51.4286 & 51.4286 \\
0.02088 & 0.00002 & 0.00075 & -0.02165
\end{array}\right]
\end{aligned}
$$

\section{References}

[1] Barlow, R.E. \& Proschan, F. (1975). Statistical Theory of Reliability and Life Testing. Probability Models. Holt, Rinehart \& Winston, New York.

[2] Esary, J. D. \& Proschan, F. (1970). A reliability bound for systems of maintained, interdependent components. J. Amer. Statist. Assoc. 65, 329-338.

[3] Funnemark, E. \& Natvig, B. (1985). Bounds for the availabilities in a fixed time interval for multistate monotone systems. Adv. Appl. Prob. 17, 638-665.

[4] Høgåsen, G. (1990). MUSTAFA programs for MUltiSTAte Fault-tree Analysis. Ph.D thesis, University of Oslo.

[5] Karlin, S. \& Taylor, H. M. (1975). A First Course in Stochastic Processes, 2nd edn. Academic Press, New York.

[6] Mørch, H. W. H. (1991). An application of multistate reliability theory on an existing network of gas pipelines. Cand. scient. thesis, University of Oslo (in Norwegian).

[7] Natvig, B. (1980). Improved bounds for the availability and unavailability in a fixed time interval for systems of maintained, interdependent components. $A d v$. Appl. Prob. 12, 200-221.

[8] Natvig, B. (1985). Multistate coherent systems, Encyclopedia of Statistical Sciences 5, 732-735.

[9] Natvig, B. (1986). Improved upper bounds for the availabilities in a fixed time interval for multistate monotone systems. Adv. Appl. Prob. 18, 577-579.

[10] Natvig, B., Sørmo, S., Holen, A. T. \& Høgåsen, G. (1986). Multistate reliability theory - a case study. Adv. Appl. Prob. 18, 921-932.

[11] Natvig, B. (1993). Strict and exact bounds for the availabilities in a fixed time interval for multistate monotone systems. Scand. J. Statist. 20, 171-175. 